\title{
Prevalencia de las alteraciones del metabolismo óseo-mineral asociadas a enfermedad renal crónica no en diálisis
}

\author{
Prevalence of bone mineral metabolism alterations associated with chronic kidney disease not on dialysis
}

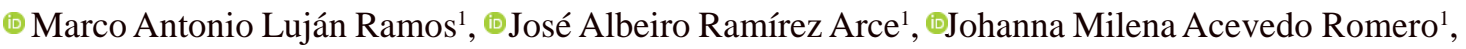 \\ (๖) Sebastián Gómez Jiménez ${ }^{1}$, ๑José Mario Cañas Osorio², ๑David Santander Bohorquez², \\ ๑๑ José Manuel Ustariz Durán ${ }^{3}$, 『 Joaquín Rodelo Ceballo ${ }^{2-4}$ \\ 'Departamento de Medicina Interna, Universidad de Antioquia, Medellín, Colombia \\ ${ }^{2}$ Departamento de Medicina Interna, Sección de Nefrología, Universidad de Antioquia, Medellín, Colombia \\ ${ }^{3}$ Programa de protección renal, Hospital General de Medellín, Colombia \\ ${ }^{4}$ Programa posgrado de Nefrología, Universidad de Antioquia, Medellín, Colombia
}

\begin{abstract}
Resumen
Introducción: la enfermedad renal crónica (ERC) es un problema de salud pública, siendo el trastorno del metabolismo óseo mineral una de sus principales complicaciones y que contribuye directamente a la morbilidad y mortalidad. Varios estudios previos han demostrado un aumento de su prevalencia a medida que disminuye la tasa de filtración glomerular (TFG), sin embargo, no contamos con datos en nuestro país ni en América Latina.

Métodos: realizamos un estudio transversal unicéntrico en un servicio de consulta de nefrología, en adultos con ERC G1 a 5 que no estuvieran en terapia de reemplazo renal, evaluados entre enero de 2014 y marzo de 2015. La recolección de datos se realizó con un instrumento predefinido que incluía datos demográficos, alteraciones de los parámetros del metabolismo mineral y óseo, y su manejo. Resultados: se incluyeron 2026 pacientes, de los cuales 1756 tenían medición de hormona paratiroidea, la edad promedio fue 74 años, el $62 \%$ eran mujeres. La distribución por grados de ERC fue: G1:4,9\%, G2:22,8 \%, G3: 57,4 \%, G4: 12,5 \% y G5:2,4 \%. Las principales causas fueron la nefropatía hipertensiva y diabética. Encontramos deficiencia de vitamina D en el 78,16 \%, hiperparatiroidismo secundario en el 63,67 \% e hiperfosfatemia en el 12,38 \%, con aumento de la prevalencia a medida que la TFG empeoraba.

Conclusiones: encontramos que las alteraciones del metabolismo mineral y óseo son frecuentes en los pacientes con enfermedad renal crónica e inician desde estadios tempranos, como se ha demostrado en otros estudios. Consideramos que estos resultados llevarán a nuevas investigaciones de manejo en pacientes con ERC.
\end{abstract}

Palabras clave: enfermedad renal crónica, hiperparatiroidismo secundario, deficiencia de vitamina D.

doi: http://dx.doi.org/10.22265/acnef.6.1.311

\begin{abstract}
Background: chronic kidney disease (CKD) is a public health problem, and bone mineral metabolism disorder is one of its main complications that directly contributes to morbidity and mortality. Several previous studies have shown an increase in its prevalence as the glomerular filtration rate (GFR) decreases, however, we do not have data from our country or Latin America.

Methods: We conducted a unicentric cross-sectional study in a nephrology consultation service in adults with CKD G1 to 5 who were not in renal replacement therapy, evaluated between January 2014 and March 2015. Data collection was performed with an instrument predefined that included demographic data, alterations of the mineral and bone metabolism parameters, and their management.

Results: 2026 patients were included, of whom 1756 had parathyroid hormone measurement, the average age was 74 years, $62 \%$ were women. The distribution by degrees of CKD was: G1: 4.9\%, G2: 22.8\%, G3: $57.4 \%$, G4: $12.5 \%$ and G5: $2.4 \%$. The main causes were hypertensive and diabetic nephropathy. We found vitamin D deficiency in $78.16 \%$, secondary hyperparathyroidism in $63.67 \%$ and hyperphosphatemia in $12.38 \%$, with an increase in prevalence as GFR worsened.

Conclusions: We found that mineral and bone metabolism alterations are frequent in patients with chronic kidney disease and start from early stages, as has been demonstrated in other studies. We believe that these results will lead to new management investigations in patients with CKD.
\end{abstract}

Key words: Renal insufficiency chronic, hyperparathyroidism, secondary, vitamin D deficiency.

doi: http://dx.doi.org/10.22265/acnef.6.1.311

Citación: Luján Ramos MA, Ramírez Arce JA, Acevedo Romero JM, Gómez Jiménez S, Cañas Osorio JM, Santander Bohorquez D, et al. Vasculitis en adultos mayores. Prevalencia de las alteraciones del metabolismo óseo-mineral asociadas a enfermedad renal crónica no en diálisis. Rev. Colomb. Nefrol. 2019;6(1):17-27. doi: http://dx.doi.org/10.22265/acnef.6.1.311

Correspondencia: Joaquín Rodelo Ceballos, joaquin.rodelo@udea.edu.co

Recibido: $20.06 .18 \cdot$ Aceptado: 29.10 .18 


\section{Introducción}

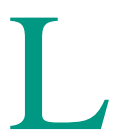

a enfermedad renal crónica (ERC) es un verdadero problema de salud pública a nivel mundial, con una alta prevalencia que aumenta a medida que envejece la población, y está asociada a diversas complicaciones, alto riesgo de mortalidad y elevados $\operatorname{costos}^{1-7}$. Una de las complicaciones más importantes y difíciles de manejar es el trastorno del metabolismo mineral y óseo, ya que este grupo de alteraciones no solo afecta la salud del hueso, también se asocia a mayor deterioro de la función renal y a un aumento de la mortalidad general, especialmente de origen cardiovascular. Uno de los componentes de este trastorno es el hiperparatiroidismo secundario, que es un trastorno multifactorial que se presenta como consecuencia de la disminución progresiva de los niveles de vitamina $\mathrm{D}$, exacerbado y perpetuado por el descenso de los niveles de calcio y la elevación progresiva de los niveles de fósforo ${ }^{8-18}$.

A medida que progresa el deterioro de la tasa de filtración glomerular, aumenta la prevalencia de las alteraciones del metabolismo mineral óseo, como nos demuestran diferentes estudios ${ }^{19-26}$. No obstante, a pesar de ser un trastorno ampliamente conocido en el contexto de la enfermedad renal crónica, las investigaciones en cuanto a la prevalencia y manejo demuestran que muchas veces es sub-diagnosticado y por tanto subtratado, e incluso en los casos en que se detecta, la instauración del manejo es compleja y con pobre respuesta metabólica.

A pesar de que conocemos datos provenientes de otras investigaciones epidemiológicas, no se han hecho estudios en Colombia, ni en América Latina, que nos permitan conocer su prevalencia. No sería prudente extrapolar los resultados sin un estudio local, debido a las diferentes características sociodemográficas entre las poblaciones. El presente estudio se realizó buscando encontrar la prevalencia de las alteraciones del metabolismo óseo-mineral, según el grado de enfermedad renal crónica en pacientes que no estuvieran en terapia de reemplazo renal en la ciudad de Medellín.

\section{Materiales y métodos}

Realizamos un estudio transversal de pacientes ingresados en el programa de protección renal en- tre enero de 2014 y marzo de 2015 en la unidad renal del Hospital General de Medellín (HGM), ubicado en la ciudad de Medellín en el departamento de Antioquia, Colombia.

Las principales variables que tuvimos en cuenta fueron: creatinina sérica para cálculo de la tasa de filtración glomerular (TFG) mediante la fórmula CKD-EPI, con lo cual se clasificaron los pacientes en los diferentes estadios de ERC (grados G1 a G5) establecidos por la guía KDIGO; además niveles de paratohormona (PTH), fósforo, calcio y 25-hidroxi vitamina D. Se definió deficiencia de vitamina D, aquella con niveles de 25 hidroxi vitamina $\mathrm{D}<30$ $\mathrm{ng} / \mathrm{dL}$, hiperfosfatemia con valor $>4,5 \mathrm{ng} / \mathrm{dL}$, hipocalcemia con valor $<8,4 \mathrm{mg} / \mathrm{dL}$ e hiperparatiroidismo un valor de PTH $>65 \mathrm{pg} / \mathrm{ml}$. Se excluyeron pacientes menores de 18 años y en terapia de reemplazo renal (hemodiálisis, diálisis peritoneal o trasplante renal).

El análisis de las variables se realizó con distribuciones de frecuencia y tabla de contingencia para las variables categóricas con la prueba $\mathrm{X}^{2}$, y para las variables continuas la distribución t de Student. Se consideró estadísticamente significativo el valor de $\mathrm{p}<0,05$. Para el análisis de correlación de variables se utilizó el coeficiente de correlación de Pearson, y para la relación vitamina D/PTH el índice de correlación de Spearman. Se realizó análisis uni y multivariado de regresión logística para desenlace de hiperparatiroidismo secundario incluyendo p >0,01 o 0,02. El análisis estadístico se realizó con el paquete estadístico Stata versión 12.

Este estudio contó con la aprobación del comité de ética del HGM, además se siguieron las normas sobre aspectos éticos de la investigación en seres humanos contenidas en la Resolución 008430 de 1993 del Ministerio de Protección Social de la República de Colombia. Los investigadores se comprometieron a respetar la confidencialidad y privacidad de la información contenida en los registros clínicos. Este trabajo no implicó intervenciones en la población de estudio (evaluación física directa, pruebas de laboratorio o aplicación de tratamiento) por lo que no les confirió riesgos a los participantes. 


\section{Resultados}

La tabla 1 muestra los datos demográficos de la población estudiada $(\mathrm{n}=2026)$, dentro de los cuales
1756 pacientes tuvieron reporte de PTH. En la distribución de variables de acuerdo a la presencia o no de hiperparatiroidismo (tabla 2), se halló una diferencia significativa en cuanto a la edad, peso, IMC,

Tabla 1. Variables demográficas totales y distribuidas según sexo.

\begin{tabular}{|c|c|c|c|}
\hline \multirow{2}{*}{ Variables } & Total & Hombres & Mujeres \\
\hline & $\mathrm{n}=\mathbf{2 0 2 6}$ & $\mathrm{n}=762(38 \%)$ & $n=1262(62 \%)$ \\
\hline \multicolumn{4}{|c|}{ Comorbilidad } \\
\hline HTA & $1868(92 \%)$ & $690(90 \%)$ & $1177(93 \%)$ \\
\hline EAOC & $82(4 \%)$ & $34(4 \%)$ & $48(4 \%)$ \\
\hline Enfermedad coronaria & $234(11 \%)$ & $106(14 \%)$ & $128(10 \%)$ \\
\hline EPOC & $329(16 \%)$ & $123(16 \%)$ & $205(16 \%)$ \\
\hline Lupus eritematosos sistémico & $25(1 \%)$ & $4(0,5 \%)$ & $21(2 \%)$ \\
\hline Diabetes mellitus & $588(29 \%)$ & $181(24 \%)$ & $407(32 \%)$ \\
\hline Carbonato calcio & $245(12 \%)$ & $78(10 \%)$ & $167(13 \%)$ \\
\hline Calcitrion & $513(26 \%)$ & $190(25 \%)$ & $323(26 \%)$ \\
\hline Paricalcitol & $24(1 \%)$ & $10(1 \%)$ & $14(1 \%)$ \\
\hline \multicolumn{4}{|c|}{ Variables de exposición } \\
\hline Edad (años) & $74 \pm 13,6$ & $74 \pm 13,6$ & $74 \pm 13,6$ \\
\hline Raza - Otra (mestizo) & $1907(95 \%)$ & $711(94 \%)$ & $1196(96 \%)$ \\
\hline Peso (Kg) & $61 \pm 12$ & $65 \pm 11$ & $58 \pm 12$ \\
\hline Talla (m) & $1.55 \pm 0,09$ & $1,63 \pm 0,08$ & $1,51 \pm 0,07$ \\
\hline IMC & $25.2 \pm 4,7$ & $24,5 \pm 3,7$ & $25,6 \pm 5,1$ \\
\hline \multicolumn{4}{|l|}{ Etiología } \\
\hline Diabetes mellitus & $371(18,3 \%)$ & $110(14 \%)$ & $261(21 \%)$ \\
\hline Hipertensión arterial & $1146(56,5 \%)$ & $419(54 \%)$ & $726(58 \%)$ \\
\hline Glomeruloesclerosis segmental y focal & $23(1,1 \%)$ & $8(1 \%)$ & $15(1 \%)$ \\
\hline Nefritis lúpica & $23(1,1 \%)$ & $5(0,7 \%)$ & $18(1,4 \%)$ \\
\hline Glomerulonefritis & $10(0,5 \%)$ & $7(0,9 \%)$ & $3(0,2 \%)$ \\
\hline Glomerulonefritis membranoproliferativa & $7(0,4 \%)$ & $2(0,3 \%)$ & $5(0,4 \%)$ \\
\hline Enfermedad renal poliquística & $16(0,8 \%)$ & $6(0,8 \%)$ & $10(0,8 \%)$ \\
\hline Nefropatía por IgA & $28(1,4 \%)$ & $11(1,4 \%)$ & $17(1,3 \%)$ \\
\hline Uropatía obstructiva & $103(5,1 \%)$ & $74(10 \%)$ & $29(2,3 \%)$ \\
\hline Desconocida & $199(9,8 \%)$ & $78(10 \%)$ & $121(10 \%)$ \\
\hline Otra & $99(4,9 \%)$ & $42(6 \%)$ & $57(5 \%)$ \\
\hline Nefrectomía por CA & $2(0,1 \%)$ & $1(0,1 \%)$ & $1(0,08 \%)$ \\
\hline Creatinina sérica $(\mathrm{mg} / \mathrm{dL})$ & $1,47 \pm 0,9$ & $1,63 \pm 0,9$ & $1,37 \pm 0,9$ \\
\hline TFG CKD-EPI (mL/min/1,73 m2) & $50,4 \pm 21.4$ & $51,7 \pm 21$ & $49,6 \pm 21$ \\
\hline PTH $(\mathrm{pg} / \mathrm{mL})$ & $79(56-116)$ & $76(55-111)$ & $80(57-119)$ \\
\hline 25 hidroxi vitamina $\mathrm{D}(\mathrm{ng} / \mathrm{mL})$ & $24,3 \pm 8,3$ & $27,1 \pm 8,3$ & $22,6 \pm 7,9$ \\
\hline Calcio (mg/dL) & $10,1 \pm 6,7$ & $10 \pm 6,2$ & $10 \pm 7,0$ \\
\hline Fósforo (mg/dL) & $3,9 \pm 0,7$ & $3,7 \pm 0,8$ & $4,0 \pm 0,6$ \\
\hline
\end{tabular}


Tabla 2. Variables distribuidas según presencia de hipotiroidismo.

\begin{tabular}{|l|c|c|c|}
\hline \multirow{2}{*}{ Variables } & Hiperparatiroidismo & $\begin{array}{c}\text { No } \\
\text { hiperparatiroidismo }\end{array}$ & \multirow{2}{*}{ Valor - P } \\
\cline { 2 - 3 } & $\mathrm{n}=1118(64 \%)$ & $\mathrm{n}=638(36 \%)$ & \\
\hline Edad & $75 \pm 13$ & $73 \pm 14$ & 0,004 \\
\hline Sexo - femenino & $706(63 \%)$ & $380(60 \%)$ & 0,131 \\
\hline Peso & $62 \pm 13$ & $60 \pm 12$ & 0,011 \\
\hline IMC & $25,6 \pm 5$ & $24,8 \pm 4$ & $<0,001$ \\
\hline HTA & $1060(95 \%)$ & $579(91 \%)$ & 0,001 \\
\hline EAOC & $52(5 \%)$ & $21(3 \%)$ & 0,17 \\
\hline Enfermedad coronaria & $138(12 \%)$ & $72(11 \%)$ & 0,513 \\
\hline EPOC & $195(17 \%)$ & $97(15 \%)$ & 0,222 \\
\hline Lupus & $7(0,6 \%)$ & $14(2,2 \%)$ & 0,004 \\
\hline Diabetes mellitus & $336(30 \%)$ & $167(26 \%)$ & 0,081 \\
\hline TFG por CKD-EPI & $43,8 \pm 18,9$ & $57,5 \pm 19,7$ & $<0,001$ \\
\hline Vitamina D & $23,6 \pm 8,3$ & $25,9 \pm 8,3$ & $<0,001$ \\
\hline Calcio & $10,2 \pm 7,3$ & $10,0 \pm 5,8$ & 0,647 \\
\hline Fósforo & $3,9 \pm 0,7$ & $3,9 \pm 0,7$ & 0,871 \\
\hline
\end{tabular}

presencia de HTA, lupus eritematoso sistémico, tasa de filtración glomerular calculada por CKD-EPI y niveles de 25 hidroxi vitamina $\mathrm{D}$. Se encontró que el $78,16 \%$ de la población estudiada presentaba deficiencia de vitamina $\mathrm{D}$, el 12,38 \% hiperfosfatemia, y el 63,67 \% hiperparatiroidismo. La distribución de dicho porcentaje de acuerdo a cada grado de ERC se muestra en la tabla 3.

Encontramos que la hipocalcemia, hiperfosfatemia, hiperparatiroidismo y deficiencia de vitamina D son más prevalentes a medida que avanzaba la disfunción renal (figura 1). El porcentaje de la población total de acuerdo al grado de deficiencia de vitamina D según cada estadio de ERC se muestra en la figura 2.

En la figura 3 se muestra la dispersión entre niveles de PTH y TFG, al igual que la relación entre niveles de 25 hidroxi vitamina D y TFG. La correlación entre edad y niveles de 25 hidroxi vitamina $\mathrm{D}$ discriminada por los diferentes estadios de ERC se muestra en la figura 4, y la correlación entre vitamina D y PTH de acuerdo al grado de ERC se muestra en la figura 5 .
En el análisis de regresión logística, teniendo en cuenta como variable dependiente el hiperparatiroidismo (tabla 4), encontramos que por cada $1 \mathrm{ml} / \mathrm{min}$ que cae la TFG por debajo de la media $(50,4 \mathrm{ml} / \mathrm{min})$, se aumenta entre 3 y $5 \%$ la prevalencia de hiperparatiroidismo secundario; además por cada $1 \mathrm{ng} / \mathrm{ml}$ de 25 hidroxi vitamina $\mathrm{D}$ por debajo de la media se aumenta de un 2 a $5 \%$ la prevalencia de hiperparatiroidismo.

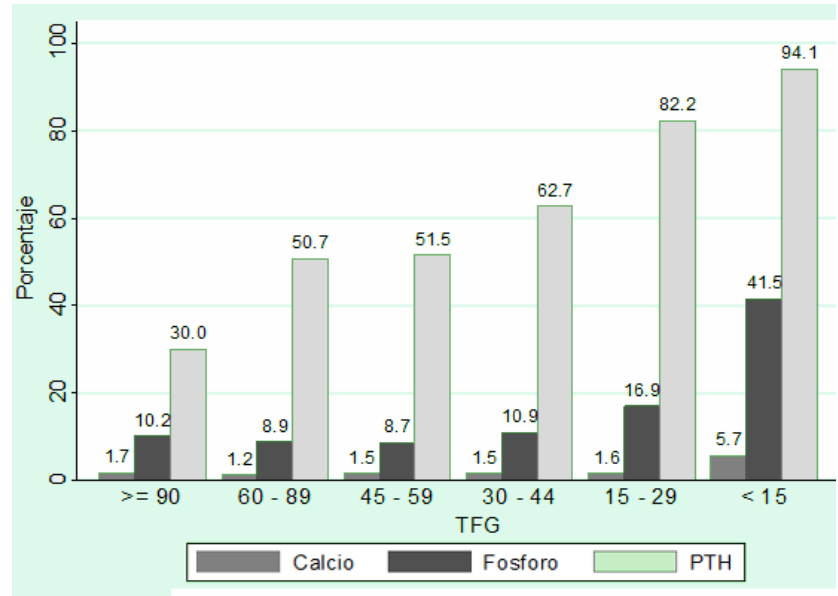

Figura 1. Prevalencia de hipocalcemia, hiperfosfatemia e hiperparatiroidismo según los grados de disfunción renal. 
Tabla 3. Distribución de complicaciones según grados de ERC.

\begin{tabular}{|l|c|c|c|c|c|c|}
\hline \multirow{2}{*}{\begin{tabular}{c}
\multirow{2}{*}{$\begin{array}{c}\text { Complicaciones } \\
\text { (\# de pacientes) }\end{array}$} \\
\cline { 2 - 7 }
\end{tabular}} & $\mathbf{6}$ & $\mathbf{G 2}$ & $\mathbf{G 3 a}$ & $\mathbf{G 3 b}$ & $\mathbf{G 4}$ & G5 \\
\cline { 2 - 7 } & $>\mathbf{9 0}$ & $\mathbf{6 0 - 8 9}$ & $\mathbf{4 5 - 5 9}$ & $\mathbf{3 0}-\mathbf{4 4}$ & $\mathbf{1 5 - 2 9}$ & $<\mathbf{1 5}$ \\
\hline Deficiencia vitamina D (1070) & $3,47 \%$ & $19,96 \%$ & $30,18 \%$ & $30,37 \%$ & $13,68 \%$ & $2,34 \%$ \\
\hline Hiperparatiroidismo (1118) & $2,24 \%$ & $14,71 \%$ & $27,26 \%$ & $33,63 \%$ & $18,39 \%$ & $3,77 \%$ \\
\hline Hiperfosfatemia (243) & $2,89 \%$ & $12,40 \%$ & $22,31 \%$ & $28,10 \%$ & $23,97 \%$ & $10,33 \%$ \\
\hline
\end{tabular}

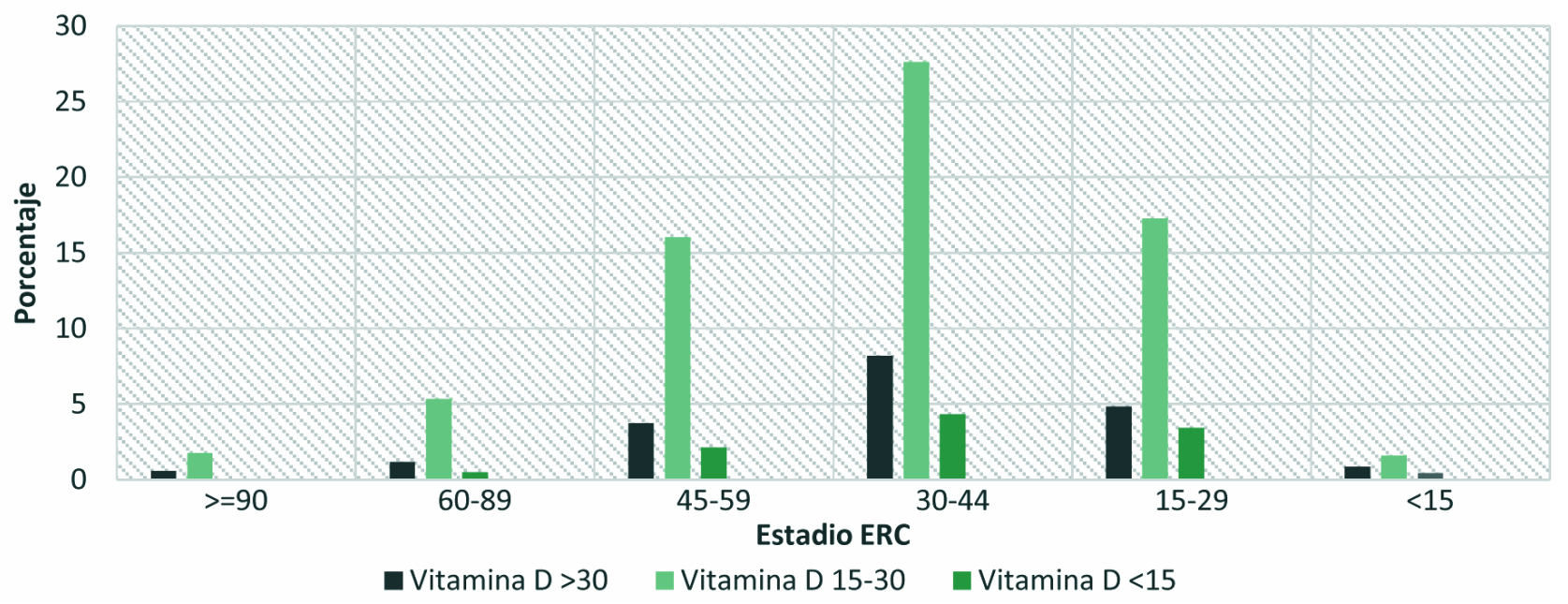

Figura 2. Niveles de vitamina D distribuidos según el grado de disfunción renal.

A

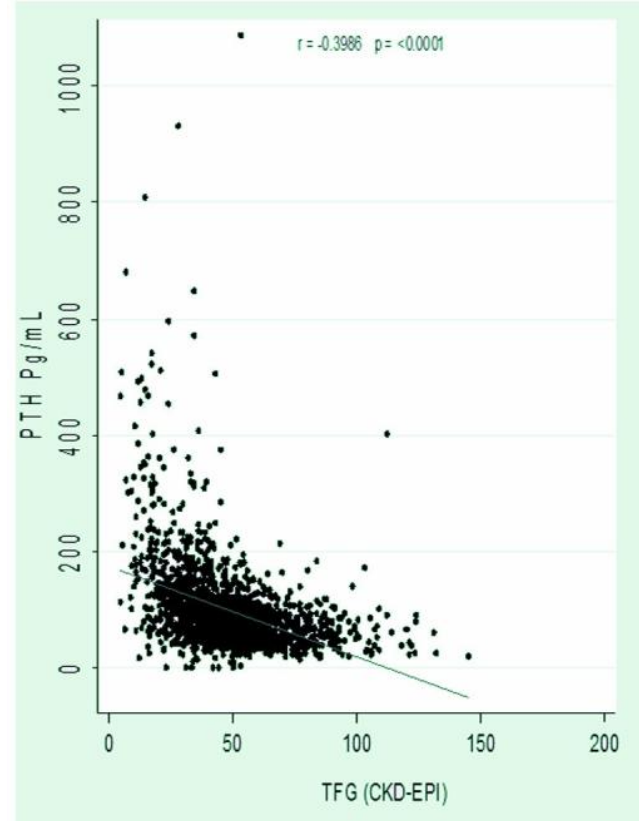

B

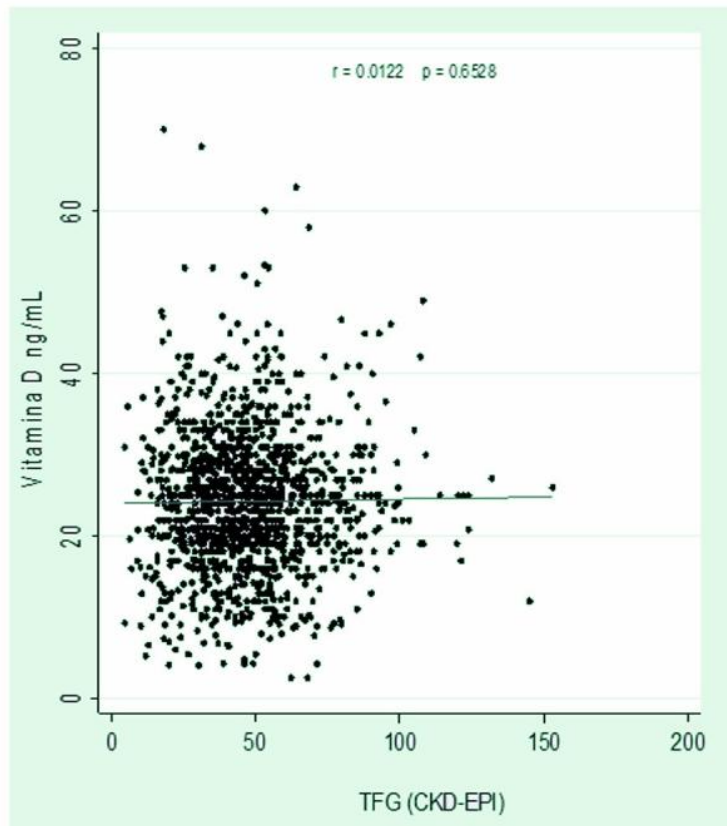

Figura 3. Gráfica de dispersión, PTH Vs TFG (A) y vitamina D Vs TFG (B). 

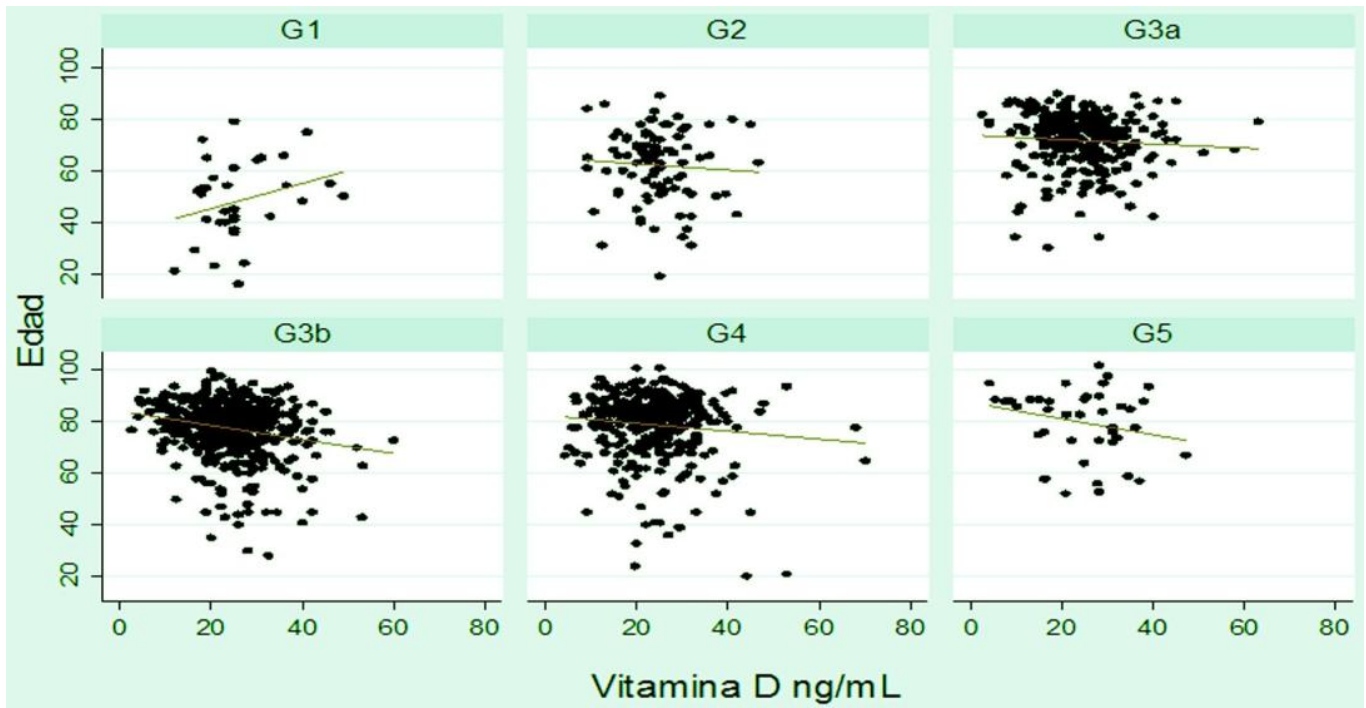

Figura 4. Correlación entre edad y vitamina D por grado de ERC.

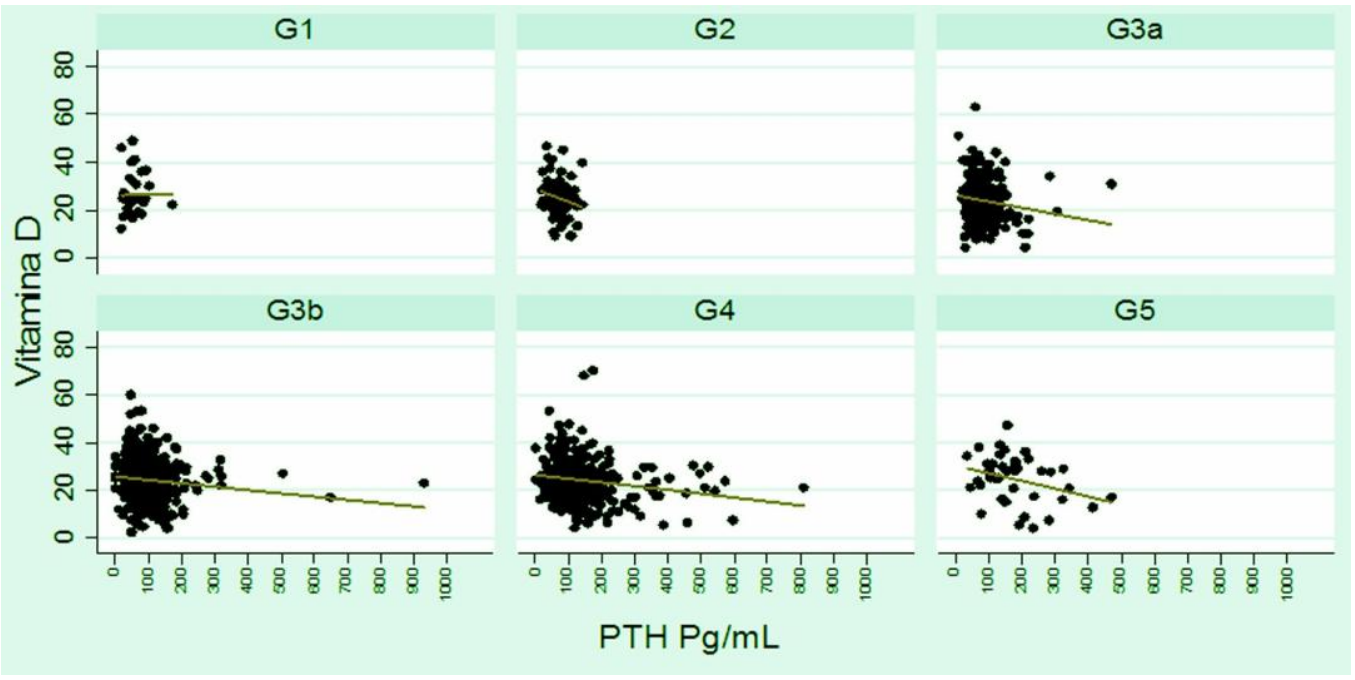

Figura 5. Correlación entre vitamina D y PTH por grado de ERC.

Tabla 4. Análisis uni y multivariado para prevalencia de hiperparatiroidismo secundario.

\begin{tabular}{|l|c|c|c|c|c|c|}
\hline \multirow{2}{*}{\multicolumn{1}{c|}{ Covariables }} & \multicolumn{3}{|c|}{ Univariado } & \multicolumn{3}{c|}{ Multivariado } \\
\cline { 2 - 7 } & OR & IC 95 \% & Valor P & OR & IC 95 \% & Valor P \\
\hline Edad & 0,99 & $0,98-0,99$ & 0,004 & 1,00 & $0,99-1,01$ & 0,354 \\
\hline Sexo & 1,17 & $0,95-1,42$ & 0,131 & 1,01 & $0,76-1,34$ & 0,930 \\
\hline TFG_CKDEPI & 1,04 & $1,03-1,04$ & 0,000 & 1,04 & $1,03-1,05$ & 0,000 \\
\hline Vitamina D ng/mL & 1,03 & $1,02-1,04$ & 0,000 & 1,04 & $1,02-1,05$ & 0,000 \\
\hline Calcio mg/dL & 1,00 & $0,98-1,01$ & 0,648 & 1,00 & $0,98-1,02$ & 0,911 \\
\hline Fosforo mg/dL & 1,01 & $0,88-1,16$ & 0,871 & 1,32 & $1,09-1,61$ & 0,004 \\
\hline Diabetes & 1,21 & $0,98-1,51$ & 0,081 & 1,04 & $0,77-1,39$ & 0,813 \\
\hline IMC & 0,96 & $0,94-0,98$ & 0,000 & 0,96 & $0,93-0,99$ & 0,007 \\
\hline
\end{tabular}

22 Enfermedad renal crónica y metabolismo óseo-mineral 


\section{Discusión}

Este estudio es el más grande publicado en Latinoamérica en su tipo; la población fue predominantemente anciana, con una edad media de 74 años y predominaron las mujeres constituyendo el $62 \%$ de la población.

La principal etiología de enfermedad renal crónica fue la hipertensión arterial con el 56,5\% de los casos, una cifra más alta que la reportada en otros estudios evaluados, y además fue la principal comorbilidad en la población independientemente de la etiología. Dentro del grupo de pacientes con hipertensión y enfermedad renal debemos tener en cuenta que también es posible que se incluyeran pacientes con cambios de nefroesclerosis asociados a la edad que contribuyeron con la alta prevalencia de enfermedad renal crónica secundaria a hipertensión arterial y pacientes con otras etiologías de la ERC que presentan hipertensión arterial como complicación. La segunda etiología más común fue la diabetes mellitus con el 18,3\% de los casos y hasta el $10 \%$ de las personas tenían etiología desconocida, que es un porcentaje importante de la población. Probablemente debido a la edad avanzada de los pacientes y las comorbilidades, las demás etiologías como las glomerulopatías representaron un porcentaje menor de los casos.

Decidimos utilizar la fórmula CKD-EPI para el cálculo de la TFG, ya que ha demostrado ser la que mejor discrimina los estadios de $\mathrm{ERC}^{27-28}$. La TFG media de la población fue $50,4 \mathrm{ml} / \mathrm{min}$, con el $21 \%$ de los pacientes encontrándose con un grado G1 y G2-A1, por lo que es probable que no tengan ninguna complicación secundaria a la enfermedad renal crónica e incluso no sería absolutamente necesario realizar seguimiento en una unidad de nefrología. La mayor parte de la población se encontraba dentro del grado $\mathrm{G} 3$, con el $57,4 \%$ de los pacientes y una menor cantidad en los grados G4 y G5 con el 12,5 \% y $2,5 \%$ respectivamente. Como no realizamos seguimiento de la función renal, no pudimos evaluar la evolución de los pacientes en cada uno de los grupos.

$\mathrm{Al}$ igual que otros autores y por el conocimiento fisiopatológico que tenemos acerca del metabolismo mineral y óseo en la enfermedad renal crónica, encontramos que a medida que se deteriora la TFG se acentuaron los cambios en los niveles de PTH, vitamina D y fósforo. Encontramos que 63,6 \% de la población cumplió los criterios de hiperparatiroidismo; incluso desde las etapas más tempranas de la enfermedad como los grados G1 y G2, entre el 30 al $50 \%$ de las personas ya tienen hiperparatiroidismo, con la mayor prevalencia en los grados G4 y G5 con el 82 y $94 \%$ respectivamente. Otros estudios ${ }^{19-22}$ demuestran un incremento progresivo de los niveles de PTH a medida que disminuye la TFG, aunque a diferencia de nuestros hallazgos la prevalencia de hiperparatiroidismo en las fases más tempranas de la enfermedad renal crónica fue menor, con rangos entre el 13 al $17 \%$ cuando la TFG era mayor a $60 \mathrm{ml} / \mathrm{min}$, como lo reportó Levin, et al ${ }^{19}$.

La elevada prevalencia de hiperparatiroidismo probablemente sea resultado en parte de la deficiencia de vitamina $\mathrm{D}$, que se encontró en el $78 \%$ de la población y el nivel fue muy similar entre los diferentes grados de enfermedad renal crónica, excepto en el G5 donde sólo el $56 \%$ presentaron niveles $<30 \mathrm{ng} / \mathrm{ml}$; aunque el valor de deficiencia $<15 \mathrm{ng} / \mathrm{ml}$ fue poco frecuente en la población y este valor si mostró una relación más directa con la caída de la TFG. A pesar de que no está claro cuál es nivel idóneo de vitamina $D$ para la supresión máxima de la PTH, datos recientes muestran que es alrededor de 27,5 a $30 \mathrm{ng} / \mathrm{ml}^{27}$. Gorriz, et al., encontró una prevalencia de hasta el $82 \%$ de deficiencia de vitamina $\mathrm{D}$, con niveles $<15 \mathrm{ng} / \mathrm{ml}$ hasta en el $32 \%$ de la población ${ }^{20}$. Pensamos que otras causas para la diferencia en cuanto al hiperparatiroidismo pudieron deberse al manejo suministrado, que no se especificó en la mayoría de estudios evaluados, y en el nuestro no evaluamos la suplementación de vitamina $\mathrm{D}$ ni el uso de medicamentos diferentes a los análogos de vitamina D para supresión de la PTH. Consideramos que los principales factores causantes de la alta prevalencia de deficiencias de vitamina D probablemente son la pobre ingesta, población anciana con menor exposición solar y la misma enfermedad renal crónica, factores, comunes en la mayoría de las poblaciones evaluadas.

El 12,3\% de la población presentó hiperfosfatemia, llamativamente incluso en el grado G1 8,7 
$\%$ la presentaron, progresando hasta una prevalencia del $16,5 \%$ y $41,5 \%$ en los que estaban en G4 y G5 respectivamente. Levin, et al., encontró una progresión muy similar de los niveles de fósforo, pero en el estadio G3a menos del $5 \%$ tenían hiperfosfatemia, lo que difiere ligeramente de nuestros datos; además los otros estudios evaluados evidenciaron que los niveles de fósforo no se elevaron sino hasta que los pacientes tuvieran una TFG $<20 \mathrm{ml} / \mathrm{min}^{19-23}$. La diferencia encontrada, aunque pequeña, pudo deberse a los medicamentos usados para el tratamiento, ya que no se evaluó el uso de quelantes de fósforo diferentes al calcio, no se discriminó el uso de calcitriol por nivel de ERC y no se realizó seguimiento; además no se consideraron otras etiologías de hiperfosfatemia.

Cuando realizamos el análisis multivariado encontramos que los factores directamente relacionados con el nivel de PTH fueron la TFG, la vitamina D y el fósforo, lo cual es esperable si conocemos el metabolismo mineral ósea en la enfermedad renal crónica. No encontramos relación con el calcio, a diferencia del estudio de Levin, et al ${ }^{19}$., y el análisis de Vassalotti, et al., de dos grandes cohortes norteamericanas $^{22}$; la falta de relación con los niveles de calcio probablemente se debió a la poca cantidad de pacientes con hipocalcemia encontrados en el grupo estudiado. Vassalotti, et al ${ }^{22}$, encontró relación del hiperparatiroidismo con la obesidad, la edad y la diabetes, datos no encontrados en nuestro estudio probablemente por la poca cantidad de personas obesas, la homogeneidad de la edad en la población, mayor peso de otras variables y los sesgos inherentes a los estudios observacionales; además no tuvimos en cuenta otras variables influyentes en este aspecto como albuminuria, los medicamentos utilizados o la raza, en cuanto a este último parámetro se ha encontrado que la prevalencia de hiperparatiroidismo es mayor en los afroamericanos.

Una de las conclusiones importantes que se pueden concluir es que una gran cantidad de pacientes tenían parámetros de $\mathrm{PTH}$, vitamina $\mathrm{D}$ y fósforo fuera de metas, según las recomendaciones actuales ${ }^{1,8,9}$ para el manejo de las alteraciones del perfil mineral y óseo, las cuales previamente fueron comentadas y a pesar de la cantidad de pacientes con hiperparatiroidismo solo $27 \%$ de los pacientes recibieran manejo con calcitriol u otro análogo de la vitamina D para su control; pero debemos tener en cuenta que no hicimos seguimiento de los pacientes para evaluar el control y que la mayoría de los datos analizados fueron medidos durante el ingreso al programa de protección renal. Aunque es un dato interesante para analizar, ya que en estudios en otras regiones muestran que la adherencia a los objetivos instaurados en general no supera el $40 \%{ }^{20-23}$, lo cual es un problema mayor, ya que al permitir que estas alteraciones persistan desde las fases iniciales, hace que posteriormente sean más complejas de controlar, perpetuando sus efectos sistémicos y como consecuencia se produce un aumento de la morbilidad y mortalidad cardiovascular ${ }^{24-27}$.

Entre las fortalezas de este estudio están el tamaño de la población evaluada, que nos permite una visión general de la enfermedad renal crónica en nuestro país y el manejo suministrado, además es una base para estudios de intervención y de seguimiento que nos ayuden a mejorar la atención que se le suministra a la población. La población fue evaluada en una unidad de protección renal de Medellín manejada por nefrólogos y las pruebas de seguimiento que se realizaron en un único centro con métodos estandarizados por lo que la variabilidad de las mediciones probablemente es mínima.

En cuanto a las debilidades, es un estudio transversal, en un único centro con datos tomados retrospectivamente, lo que irremediablemente introduce sesgos e imprecisiones en las medidas; además tampoco se pueden generalizar los datos a toda la población con enfermedad renal que no se sigue en unidades de nefrología. Faltaron datos importantes como discriminar de forma más certera la raza de los pacientes, aunque la población predominante era mestiza; no se evaluó la suplencia de vitamina $\mathrm{D}$, el uso de quelantes de fósforo diferentes al calcio, ni el uso de cinacalcet o la distribución de los medicamentos según los grados de ERC. Tampoco se evaluaron los resultados según las diferentes causas de enfermedad renal crónica, aunque en general hubo una baja prevalencia de enfermedades glomerulares y no se determinaron otras etiologías como nefritis intersticial, toxicidad por medicamentos, secuelas de 
enfermedad renal aguda o enfermedades vasculares renales, en parte por una limitación inicial en el listado del formulario de recolección, la menor prevalencia y la dificultad de realizar el diagnóstico.

En conclusión, observamos que las alteraciones del metabolismo mineral óseo en el paciente con enfermedad renal crónica son frecuentes y progresivas a medida que avanza la disfunción renal, y su manejo en muchos casos es inadecuado con el riesgo que esto conlleva. Nuestros resultados fueron similares a los de otras cohortes publicadas y de pie para estudios de manejo y seguimiento para evaluar la calidad y efectividad de los servicios suministrados a los pacientes.

\section{Conflicto de intereses}

Los autores no tienen los posibles conflictos de interés.

\section{Financiación}

Los autores han declarado no tener financiación y no recibir ningún apoyo económico.

Se recibió apoyo logístico del centro de ensayos clínicos del Hospital General de Medellín.

\section{Responsabilidades éticas}

\section{Protección de personas y animales}

Los autores declaran que para esta investigación no se han realizado experimentos en seres humanos ni en animales.

\section{Confidencialidad de los datos}

Los autores declaran que han seguido los protocolos de su centro de trabajo sobre la publicación de datos de pacientes.

\section{Derecho a la privacidad y consentimiento in- formado}

Los autores declaran que en este artículo no aparecen datos de pacientes.

\section{Contribución de los autores}

Investigador principal: Marco Luján, José Ramírez.

Coinvestigador: Johanna Acevedo, Sebastián Gómez, José Cañas, David Santander, José Ustariz.

Director de grupo de investigación; asesor: Joaquín Rodelo. 


\section{Rev. Colomb. Nefrol. 2019;6(1): 17 - 27, enero - junio de 2019 http://www.revistanefrologia.org}

http://dx.doi.org/10.22265/acnef.6.1.311

\section{Referencias}

1. Martínez-Castelao A, Górriz-Teruel JL, Bover-Sanjuán J, Segura-de la Morena J, Cebollada J, Escalada J, et al. Documento de consenso para la detección y manejo de la enfermedad renal crónica. Nefrología. 2014; 34(2):243-62.

2. Kidney Disease: Improving Global Outcomes (KDIGO) CKD-MBD Work Group. KDIGO clinical practice guideline for the diagnosis, evaluation, prevention, and treatment of Chronic Kidney Disease-Mineral and Bone Disorder (CKD-MBD). Kidney Int Suppl. 2009;2(113):S1-130.

3. Jha V, Garcia-Garcia G, Iseki K, Li Z, Naicker S, Plattner B, et al. Chronic kidney disease: Global dimension and perspectives. Lancet. 2013;382(9888):260-72. https://doi.org/10.1016/S0140-6736(13)60687-X

4. Sociedad Colombiana de Nefrología e Hipertensión. Registro colombiano de diálisis y trasplante. 2007.

5. Fondo Colombiano de Enfermedades de Alto Costo. Enfermedad renal crónica, hipertensión arterial y diabetes mellitus. $2015 ; 152$. From: https://cuentadealtocosto.org/site/images/Situación_de_la_Enfermedad_Renal_Crónica_en_Colombia_2015.pdf

6. Gansevoort RT, Correa-Rotter R, Hemmelgarn BR, Jafar TH, Heerspink HJL, Mann JF, et al. Chronic kidney disease and cardiovascular risk: Epidemiology, mechanisms, and prevention. Lancet. 2013;382(9889):339-52. https://doi.org/10.1016/S0140-6736(13)60595-4

7. Tonelli M, Wiebe N, Culleton B, House A, Rabbat C, Fok M. Chronic Kidney Disease and Mortality Risk: A Systematic Review. J Am Soc Nephrol. 2006;17(7):2034-47. https://doi.org/10.1681/ASN.2005101085

8. Eknoyan G, Levin A, Levin NW. Bone metabolism and disease in chronic kidney disease. Am J Kidney Dis. 2003;42(4):1-201.

9. Kidney Disease: Improving Global Outcomes (KDIGO) CKD-MBD Work Group. KDIGO clinical practice guideline for the diagnosis, evaluation, prevention, and treatment of Chronic Kidney Disease-Mineral and Bone Disorder (CKD-MBD). Kidney Int Suppl. 2009;2(113):S1-130.

10. Cunningham J, Locatelli F, Rodriguez M. Secondary hyperparathyroidism: Pathogenesis, disease progression, and therapeutic options. Clin J Am Soc Nephrol. 2011;6(4):913-21. https://doi.org/10.2215/CJN.06040710

11. Mac Way F, Lessard M, Lafage-Proust MH. Pathophysiology of chronic kidney disease-mineral and bone disorder. Jt Bone Spine. 2012;79(6):544-9. https://doi.org/10.1016/j.jbspin.2012.09.014

12. Shirley DG, Unwin RB. Renal physiology. Comprehensive clinical nephrology. 4th edition. Floege J, Johnson RJ, Feehally J. Elseviers Saunders, St Louis, 2010.

13. Mejía N, Roman-García P, Miar AB, Tavira B, Cannata-Andía JB. El complejo escenario de las alteraciones de metabolismo óseo y mineral en la enfermedad renal crónica. Nefrologa. 2011;31(5): 514-9. https://doi.org/10.3265/Nefrologia.pre2011.Jun.10926

14. Hruska KA, Seifert M, Sugatani T. Pathophysiology of the chronic kidney disease - Mineral bone disorder. Curr Opin Nephrol Hypertens. 2015;24(4):303-9. https://doi.org/10.1097/MNH.0000000000000132

15. Martin KJ, Gonzalez EA. Metabolic Bone Disease in Chronic Kidney Disease. J Am Soc Nephrol. 2007;18(3):875-85. https://doi.org/10.1681/ASN.2006070771

16. Block GA, Klassen PS, Lazarus JM, Ofsthun N, Lowrie EG, Chertow GM. Mineral metabolism, mortality, and morbidity in maintenance hemodialysis. J Am Soc Nephrol. 2004;15(8):2208-18. https://doi.org/10.1097/01.ASN.0000133041.27682.A2

17. Anderson JL, Vanwoerkom RC, Horne BD, Bair TL, May HT, Lappé DL, et al. Parathyroid hormone, vitamin D, renal dysfunction, and cardiovascular disease: Dependent or independent risk factors? Am Heart J. 2011;162(2):331-339.e2. https://doi.org/10.1016/ j.ahj.2011.05.005

18. Aly Z Al, González EA, Martin KJ, Gellens ME. Achieving K/DOQI laboratory target values for bone and mineral metabolism: An uphill battle. Am J Nephrol. 2004;24(4):422-6. https://doi.org/10.1159/000080087

19. Levin A, Bakris GL, Molitch M, Smulders M, Tian J, Williams LA, et al. Prevalence of abnormal serum vitamin D, PTH, calcium, and phosphorus in patients with chronic kidney disease: Results of the study to evaluate early kidney disease. Kidney Int. 2007;71(1):318. https://doi.org/10.1038/sj.ki.5002009

20. Górriz JL, Molina P, Bover J, Barril G, Martín-de Francisco ÁL, Caravaca F, et al. Characteristics of bone mineral metabolism in patients with stage 3-5 chronic kidney disease not on dialysis: Results of the OSERCE study. Nefrologia. 2013;33(1):46-60. https://doi.org/10.3265/Nefrologia.pre2012.Nov.11703

26 Enfermedad renal crónica y metabolismo óseo-mineral 
21. Craver L, Marco MP, Martínez I, Rue M, Borràs M, Martín ML, et al. Mineral metabolism parameters throughout chronic kidney disease stages 1-5 - Achievement of K/DOQI target ranges. Nephrol Dial Transplant. 2007;22(4):1171-6. https://doi.org/10.1093/ $\mathrm{ndt} / \mathrm{gfl} 718$

22. Vassalotti JA, Uribarri J, Chen SC, Li S, Wang C, Collins AJ, et al. Trends in Mineral Metabolism: Kidney Early Evaluation Program (KEEP) and the National Health and Nutrition Examination Survey (NHANES) 1999-2004. Am J Kidney Dis. 2008;51(4 SUPPL. 2):1999-2004. https://doi.org/10.1053/j.ajkd.2007.12.018

23. Hoy T, Fisher M, Barber B, Borker R, Stolshek B, Goodman W. Adherence to K/DOQI practice guidelines for bone metabolism and disease. Am J Manag Care. 2007 Nov;13(11):620-5.

24. Ramos AM, Albalate M, Vazquez S, Caramelo C, Egido J, Ortiz A. Hyperphosphatemia and hyperparathyroidism in incident chronic kidney disease patients. Kidney Int Suppl. 2008 Dec;(111):S88-93. https://doi.org/10.1038/ki.2008.543

25. Kestenbaum B, Katz R, De Boer I, Hoofnagle A, Sarnak MJ, Shlipak MG, et al. Vitamin D, parathyroid hormone, and cardiovascular events among older adults. J Am Coll Cardiol. 2011;58(14):1433-41. https://doi.org/10.1016/j.jacc.2011.03.069

26. Covic A, Kothawala P, Bernal M, Robbins S, Chalian A, Goldsmith D. Systematic review of the evidence underlying the association between mineral metabolism disturbances and risk of all-cause mortality, cardiovascular mortality and cardiovascular events in chronic kidney disease. Nephrol Dial Transplant. 2009;24(5):1506-23. https://doi.org/10.1093/ndt/gfn613

27. Durazo-Arvizu R a, Dawson-Hughes B, Sempos CT, Yetley E a, Looker AC, Cao G, et al. Three-phase model harmonizes estimates of the maximal suppression of parathyroid hormone by 25-hydroxyvitamin D in persons 65 years of age and older. J Nutr. 2010;140(3):595-9. https://doi.org/10.3945/jn.109.116681

28. Mahmoodi BK. Comparison of Risk Prediction Using the CKD-EPI Equation and the MDRD Study Equation for Estimated Glomerular Filtration Rate. JAMA J Am Med Assoc. 2012;307(18):1941. https://doi.org/10.1001/jama.2012.3954. 